\title{
Mobile Application and Wearable Sensors for Use in Cognitive Behavioral Therapy for Drug Addiction and PTSD
}

\author{
Richard Ribon Fletcher, Member IEEE, Sharon Tam, Olufemi Omojola, \\ Richard Redemske, Szymon Fedor, Joseph Mugisha Moshoka
}

The Media Laboratory, Massachusetts Institute of Technology, Cambridge, MA 02139

\begin{abstract}
We present a mobile system for cognitive behavioral therapy (CBT) developed for an ongoing study for patients with drug-addiction and post-traumatic stress disorder (PTSD). The mobile platform consists of two parts: a wearable sensor system for collecting algorithm training data in the lab, and a mobile phone application used to deliver therapeutic interventions as triggered by real-time sensor data. Ecological momentary assessments (EMA) are also used as a means of collecting subjective data and validating the sensor classification algorithm. We provide a brief description of the wearable sensors, mobile phone software and network architecture used in the study.
\end{abstract}

Index Terms - mobile health, cognitive behavioral therapy, wearable sensors, drug addiction, PTSD.

\section{INTRODUCTION AND MOTIVATION}

\section{A. The Need for Mobile Monitoring and Interventions}

Mental health and behavioral disorders are generally difficult to treat. Common examples include a variety of addictions (drugs, gambling, food, etc.) as well as anxiety disorders. Psychiatric interventions traditionally occur in the clinic, through scheduled doctor visits. Unfortunately, due to social stigma, financial means, or inconvenience, patients often discontinue the doctor visits before the treatment is complete. In addition, prescribing medication may not be appropriate or desired by the patient.

Mobile technologies thus represent an opportunity to be able to monitor patient health in between doctor visits and also provide an additional therapeutic channel to the patient.

\section{B. Ecological Momentary Assessments (EMA)}

Ecological momentary assessments (EMA) delivered on mobile devices have become a standard way of capturing information from the patient in a natural setting, without the need for recall or patient diaries.[1]

While these EMA surveys are very useful in capturing subjective information such as the patent's mood, it requires the person's participation and attention. For example, a person with anxiety disorder may have an anxiety attack while driving or when encountering a crowd of people, but these moments may be completely
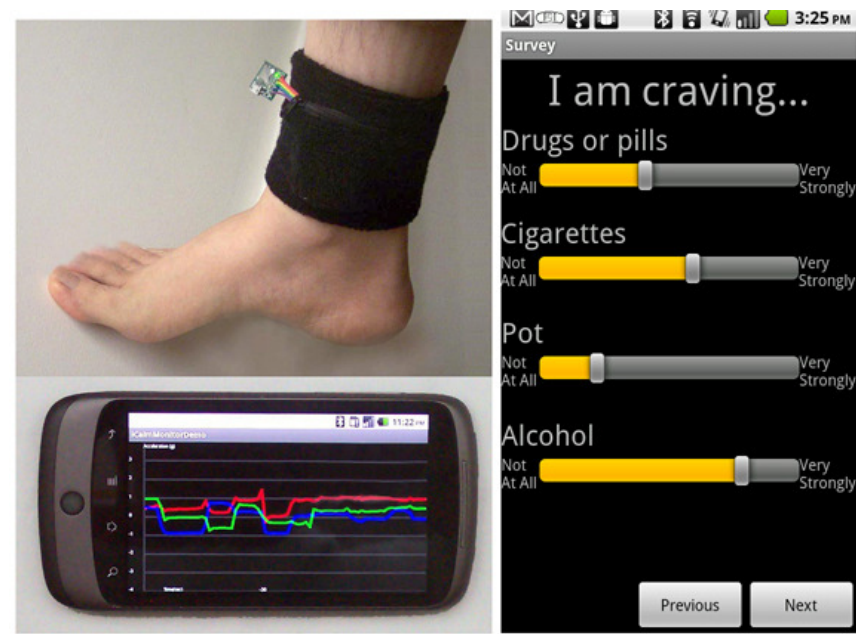

Fig. 1. (top) Photograph of ankle sensor band; (bottom) display of real-time physiology data; and (right) sample EMA screen on mobile phone. Intervention message not shown.

inappropriate for answering an EMA survey. As a result, this useful information and correlation is missed.

Wearable sensors thus provide an opportunity to monitor a person's physical and emotional state automatically and continuously.

\section{Cognitive Behavioral Therapy (CBT)}

Cognitive behavioral therapy (CBT) is commonly used for treating many mental health disorders such as treatment of addictions or anxiety.[2] The therapy consists of techniques for self reflection as well as techniques for coping with episodes of craving or stress. Examples include relaxation techniques (e.g. breathing exercises) or imagery ("imagine yourself on a beach.."). Over the past decade, CBT interventions have been delivered via the Internet (web sites) and, more recently, via PDA's and mobile phones.

Unfortunately, CBT suffers from similar practical issues as EMA, which is that the mobile phone or PDA does not know the person's physical or emotional state and thus relies on the person to request the intervention or simply schedules the intervention at various times of the day.

To address this issue, new approaches to CBT have emerges that enable "just in time" interventions, that are 
delivered at the appropriate time when the patient needs it.[3] We present here a mobile system for delivering CBT that is currently being used in a patient study.

\section{MOBILE CBT PLATFORM}

The basic elements of our system are described below.

\section{A. Wearable Sensor Bands}

As shown in Figure 1, the wearable sensor consists of a neoprene band that contains circuitry for measuring electrodermal activity (EDA), 3-axis motion, and temperature. Although this device could be worn on the wrist, the patients in our focus group preferred the ankle for reasons of comfort as well as for privacy.

The sensor bands contain a low-power microcontroller (Atmel XMega) with 12-bit ADC resolution for the analog sensors. A Bluetooth radio with low-power protocol was incorporated into the electronic module to communicate directly with the mobile phone. A 2 GB MicroSD card is also included in the band to provide local storage and caching of data when the phone is not in range. The sensor band also contains a $500 \mathrm{mAh}$ battery which provides 9 hours of continuous use at $4 \mathrm{~Hz}$ sampling rate and Bluetooth transmission rate. Longer battery life is possible with slower sampling rate and shorter duty cycle.

The sensor sampling rate is adjustable in real-time, but a fixed $8 \mathrm{~Hz}$ sampling rate was used for data collection in the lab and $4 \mathrm{~Hz}$ for the field deployment. During use, the sensor band module does some local analysis of the data then the phone applies a classification algorithm to decide whether or not to trigger an intervention message to be displayed.

\section{B. Mobile Phone}

The mobile phone software was implemented using Android OS and the JAVA SDK. Two separate phone models were used: the Nexus One phone, running Android 2.2 for use in the clinical data collection by the research staff, and the compact Motorola Flipout phone, running Android 2.1, which was suitable for use a dedicated CBT device to be carried by the patient.

Two separate phone applications were created for use in the study. For the purpose of collecting training data in the clinic, we created an application that is capable of recording live data from multiple Bluetooth sensor bands simultaneously and displaying live plots (Fig. 1). For the purpose of mobile CBT, a multi-threaded application was created which runs several services in the background and launches Android activities to display EMA surveys (Fig. 1) and intervention messages. All EMA surveys were followed by an appropriate empathetic message based on the user's input. Our main program was implemented as a custom home screen activity, which launches when the phone is powered on, and also disables all other user buttons and controls so that the phone could not be used for any other purpose other than CBT.

\section{Web Server and Database}

A custom server JAVA application was created to support remote log-in and review of anonymous deidentified patient data and user settings. In addition to viewing patient sensor data and assessment results, a key feature was the means to remotely update and adjust the CBT sensor algorithm parameters on each patient's phone. Patient data was stored on a remote Microsoft MS-SQL secure database server located in a high-security facility requiring VPN access.

\section{PATIENT STUDY}

The mobile CBT platform was developed as part of an ongoing study with military veterans that are recovering from drug addition and PTSD, which have emerged as significant risk factors for homelessness, family issues, and suicides. (NIH project 5RC1DA028428-02) Patients were recruited from the resident drug rehabilitation program at the local Veterans Administration Hospital. The study protocol consisted of several sessions in the clinic to acquire baseline data followed by 4-8 weeks of CBT using the mobile phone. Most patients tested thus far have exhibited at least some degree of reactivity (EDA and heart rate response) when presented with PTSD and drug cues in the lab. Preliminary patient feedback on the mobile CBT has been positive. This work is ongoing.

\section{DEMONSTRATION}

For Pervasive Health 2011, we shall have a live demonstration of our mobile CBT application as well as a demonstration of live data collection that we use for cue testing in the clinic. We shall also report on the recent findings and lessons learned from our study.

\section{REFERENCES}

[1] S. Stone, S.Shiffman; "Ecological Momentary Assessment (EMA) in Behavioural Medicine," Ann. Behav. Med. 16, pp. 199-202 (1994).

[2] T.M. Erickson, M.G. Newman; "Cognitive Behavioral Psychotherapy for Generalized Anxiety Disorder: a Primer," Expert Rev. Neurotherapeutics 5(2), pp. 247-257 (2005).

[3] M. Morris, F. Guilak, "Mobile Heart Health:Project Highlight," IEEE Pervasive Computing. vol. 8; no.2 , pp. 57-61, April-June 2009. 